\title{
THE GROWTH OF CERTAIN AMALGAMATED FREE PRODUCTS AND HNN-EXTENSIONS
}

\author{
M. EDJVET and D. L. JOHNSON
}

(Received 23 March 1990; revised 3 August 1990)

Communicated by $\mathbf{H}$. Lausch

\begin{abstract}
Here we mean growth in the sense of Milnor and Gromov. After a brief survey of known results, we compute the growth series of the groups

$$
\left\langle x, y \mid y^{-1} x^{p} y=x^{p}\right\rangle \text { and }\left\langle x, y \mid x^{p}=y^{p}\right\rangle, p \geq 2,
$$

with respect to generators $\{x, y\}$. This is done using minimal normal forms obtained by informal use of judiciously chosen rewrite rules. In both of these examples the growth series is a rational function, and we suspect that this is not the case for the Baumslag-Solitar group

$$
\left\langle x, y \mid y^{-1} x^{2} y=x^{3}\right\rangle \text {. }
$$
\end{abstract}

1991 Mathematics subject classification (Amer. Math. Soc.): 20 E 06.

\section{Introduction}

Here we mean growth in the sense of Milnor [14]. Thus, with a group $G$ generated by a finite set $X$, we associate the number $c_{n}$ of elements of $G$ that can be written as a product of $n$ elements of $X^{ \pm}$and no fewer. Examples of $(G, X)$ for which the $c_{n}$, or equivalently, the growth series

$$
\gamma(t)=\sum_{n \geq 0} c_{n} t^{n}
$$

have been calculated exactly are the fundamental group of the Klein bottle [14], free products [2], the (first) discrete Heisenberg group [1], closed surface

(C) 1992 Australian Mathematical Society 0263-6115/92 \$A2.00+0.00 
groups and compact hyperbolic groups [3], certain torus knot groups [15], [2], and certain wreath products [11], [9]. Following a suggestion made to one of us by R. C. Lyndon in March 1988, we extend this list by computing the growth of the groups

$$
\left\langle x, y \mid y^{-1} x^{p} y=x^{p}\right\rangle, \quad\left\langle x, y \mid x^{p}=y^{p}\right\rangle,
$$

for all $p \geq 2$, with respect to $X=\{x, y\}$, in Sections 4,5 , respectively.

The method (see Section 1 and also [9]) is crude: merely count the words of each length in a minimal normal form for $G$ (with respect to $X$ ), obtained by informal use of judiciously chosen rewrite rules ([10], [6]). The lemmas needed for this are described in Section 2. They generalize the method for free products used in [2] (which also contains calculations of the growth of free products with finite and central amalgamation, groups with one end, certain torus knot groups, and a class of one-relator groups), which has its origin in [4], and similar techniques are to be found in [9]. The whole process is illustrated in Section 3 in the case of the group $\left\langle x, y \mid y^{-1} x^{2} y=x^{2}\right\rangle$.

It turns out that the groups considered are well-behaved in the sense that their growth series are rational functions. Examples of $G, X$ with $\gamma(t)$ irrational are scarce: other than groups with insoluble word problem [3], groups of intermediate growth [5], [8], and the second discrete Heisenberg group [7], we know of none. We have carried out substantial but inconclusive calculations on each of the groups $\left\langle x, y \mid y^{-1} x y=x^{2}\right\rangle$, following a suggestion of D. J. Collins, and $\left\langle x, y \mid y^{-1} x^{2} y=x^{3}\right\rangle$, the Baumslag-Solitar group, and suspect that the first has rational growth and that the second has not.

The fact that the growth functions of the three examples listed above have proved to be quite elusive may seem somewhat surprising at first. The difficulty with HNN extensions and amalgamated free products is that the usual normal forms (as defined, for example, in [12, Chapter IV]) are not minimal forms in general.

\section{The method}

The method is purely combinatorial: let $G$ be the group in hand, $X=$ $\{x, y\}, F$ the free group on $X, \nu: F \rightarrow G$ the natural map, and $l(w)$ the usual length of $w \in F$ as a word in $X^{ \pm}$. Then we describe a set $\mathscr{R}=\{f$ : $F \rightarrow F$ \} of rewrite rules with the following properties:

(i) for all $w \in F$ and $f \in \mathscr{R}, f(w) \nu=w \nu$;

(ii) for all $w \in F$ and $f \in \mathscr{R}, l(f(w)) \leq l(w)$;

(iii) the set $T=\{w \in F \mid f(w)=w, \forall f \in \mathscr{R}\}$ of irreducibles forms a transversal for $\operatorname{Ker} \nu$ in $F$. 
Regarding $f \in \mathscr{R}$ as a rule $u r v \rightarrow u s v$, (i) holds provided $r=s$ is a relation in $G$, and (ii) holds if $l(s) \leq l(r)$. (Rules such as $u x x^{-1} v \rightarrow u v$ may be included in $\mathscr{R}$ or, alternatively, we may work with reduced words only.) Then (iii) will hold if we can establish uniqueness, that is, for distinct elements $t, t^{\prime} \in T, t \nu \neq t^{\prime} \nu$. It follows from (i) and (ii) that $T$ is a minimal normal form for $G$, that is, if $w \in T$ and $w^{\prime} \in F$ with $w \nu=w^{\prime} \nu$, then $l(w) \leq l\left(w^{\prime}\right)$, and the growth coefficient $c_{n}$ of $G$ is just the number of words of length $n$ in $T$.

\section{Counting lemmas}

Let $H$ be a subset of a group $G$ and suppose that with respect to a generating set $Y$ of $G, H$ has growth series which we denote by $\gamma_{H}$.

Let $z$ be a letter not in $G$ and $\Lambda$ the set of words of the form

$$
z^{\varepsilon_{1}} v_{1} \ldots z^{\varepsilon_{n}} v_{n} \quad(n \geq 1) \quad\left(\varepsilon_{i}= \pm 1,1 \leq i \leq n\right)
$$

where each $v_{i}(1 \leq i \leq n)$ is a word in $Y$ belonging to $H$, and if $v_{j}=e$ for some $j, 1 \leq j<n$, then $\varepsilon_{j}$ and $\varepsilon_{j+1}$ have the same sign. Then standard combinatorial arguments show that for $k \geq 1$ the number of elements of length $k$ in $\Lambda$ with respect to $\{z, Y\}$ is the coefficient of $t^{k}$ in

$$
P_{\Lambda}(t)=\sum_{n \geq 0}\left[\left(2 t\left(\gamma_{H}-1\right)\right)^{n}\left(\sum_{i \geq 0}\left(2\left(\begin{array}{c}
n+i \\
i
\end{array}\right)-\left(\begin{array}{c}
n+i-1 \\
i
\end{array}\right)\right) t^{i}\right)\right]
$$

where $n+i>0$. In fact we have the following result.

LEMMA 1. The growth series $\gamma_{\Lambda}(t)$ of $\Lambda$ with respect to $\{z, Y\}$ is given by

$$
\gamma_{\Lambda}(t)=\frac{1+t}{1+t-2 t \gamma_{H}}
$$

Proof. We have using the fact that for $i, n \neq 0$,

$$
2\left(\begin{array}{c}
n+i \\
i
\end{array}\right)-\left(\begin{array}{c}
n+i-1 \\
i
\end{array}\right)=\left(\begin{array}{c}
n+i \\
i
\end{array}\right)+\left(\begin{array}{c}
n+i-1 \\
i-1
\end{array}\right)
$$


that

$$
\begin{aligned}
& \gamma_{\Lambda}(t)=1+P_{\Lambda}(t) \\
& =1+2 \sum_{j \geq 1} t^{j}+\sum_{n \geq 1}\left[\left(2 t\left(\gamma_{H}-1\right)\right)^{n}\left(1+\sum_{i \geq 1}\left(\left(\begin{array}{c}
n+i \\
i
\end{array}\right)+\left(\begin{array}{c}
n+i-1 \\
i-1
\end{array}\right)\right) t^{i}\right)\right] \\
& =1+2 \sum_{j \geq 1} t^{j}+\sum_{n \geq 1}\left[\left(2 t\left(\gamma_{H}-1\right)\right)^{n}\left(1+\left(\frac{1}{(1-t)^{n+1}}-1\right)+\frac{t}{(1-t)^{n+1}}\right)\right] \\
& =1+\frac{2 t}{1-t}+\frac{1+t}{1-t} \sum_{n \geq 1} \frac{\left(2 t\left(\gamma_{H}-1\right)\right)^{n}}{(1-t)^{n}}
\end{aligned}
$$

and the result follows.

Now let $X=\{x, y\}$ and for $A, B \subseteq F(X) \backslash\{e\}$ let $A \pi B$ denote the set of alternating products

$$
y_{0}\left(\prod_{i=1}^{n} x_{i} y_{i}\right) x_{n+1} \quad(n \geq 0),
$$

where $y_{0} \in B \cup\{e\}, x_{n+1} \in A \cup\{e\}$ and $x_{i} \in A, y_{i} \in B \quad(1 \leq i \leq n)$.

We write $A \pi_{r} B, A_{r} \pi B$ (respectively) to denote those words in $A \pi B$ which are either trivial or end, begin (respectively) with a member of $B$. The subsets $A \pi_{l} B$ and $A_{l} \pi B$ are defined similarly. Further, $A_{r} \pi_{r}^{*} B$ denotes those words in $(A \pi B) \backslash\{e\}$ which both begin and end with members of $B$, and $A_{l} \pi_{r} B$ denotes those words in $A \pi B$ which begin with a member of $A$ and end with a member of $B$. So, for example, $A \pi_{r} B=A_{r} \pi_{r}^{*} B \cup A_{l} \pi_{r} B$.

We give the next result without proof (see [2] or [9]).

LEMMA 2. (i) If $A, B$ (respectively) has growth series $\gamma_{A}(t), \gamma_{B}(t)$ (respectively) with respect to $X$ then the growth series of $A \pi B$ with respect to $X$ is

$$
\gamma(t)=\frac{\left(1+\gamma_{A}(t)\right)\left(1+\gamma_{B}(t)\right)}{1-\gamma_{A}(t) \gamma_{B}(t)}
$$

(ii) The growth series of $A \pi_{r} B$ is

$$
\gamma_{r}(t)=\frac{1+\gamma_{B}(t)}{1-\gamma_{A}(t) \gamma_{B}(t)}
$$

\section{An example}

We take the presentation

$$
G=\left\langle x, y \mid y^{-1} x^{2} y=x^{2}\right\rangle
$$


and calculate the growth series $\gamma(t)$ of $G$ with respect to $X=\{x, y\}$.

Starting with an arbitrary reduced word in $F(X)$, we apply the following rewrite rules in turn. (At any stage, if we can freely reduce then we do so.)

RR1 Collect any power of the central element $x^{2}$ to the left.

RR2 Replace a word $\left(u x^{2} v x^{-1} w\right)^{ \pm 1},\left(u x^{-1} v x^{2} w\right)^{ \pm 1}$ (respectively) by $(u v x w)^{ \pm 1},(u x v w)^{ \pm 1}$ (respectively).

RR3 Replace a word $u x^{-1} v x w$ by $u x v x^{-1} w$.

Thus our proposed set of normal forms $T$ consists of all (freely reduced) words belonging to the following list.

(1) $x^{\alpha}$ where $\alpha \in \mathbb{Z}$.

$(2)^{ \pm}\left(x^{\beta} y^{\varepsilon_{1}} x^{\mu_{1}} \ldots y^{\varepsilon_{n}} x^{\mu_{n}}\right)^{ \pm 1}$, where $\beta \geq 0, \varepsilon_{i}= \pm 1, \mu_{i}=0$ or $1(1 \leq$ $i \leq n)$ and $n \geq 1$.

(3) $x^{\mu_{1}} y^{\varepsilon_{1}} \ldots x^{\mu_{n}} y^{\varepsilon_{n}} x y^{\mu} x^{-1} y^{\delta_{1}} x^{\nu_{1}} \ldots y^{\delta_{m}} x^{\nu_{m}}$, where $\mu \neq 0, n, m \geq 0$, $\varepsilon_{i}= \pm 1, \mu_{i}=0$ or $1(1 \leq i \leq n), \delta_{j}= \pm 1, \nu_{j}=0$ or -1 $(1 \leq j \leq m)$.

In fact, $I=\left\{y^{\mu}: \mu \in \mathbb{Z} \backslash\{0\}\right\}$ is a subset of $(2) \cap(2)^{-1}$ and so we must subtract a copy of this to ensure uniqueness. Observe that each freely reduced element in the list is a reduced word in the HNN sense and so each normal form of type (1) is distinct from those of type (2) ${ }^{ \pm 1}$ and (3). Furthermore, if $g=h$ in $G$ then the exponent sum of $y$ in $g h^{-1}$ is zero; and (3) is self-inverse. All this reduces the cases that need be considered to

(i) $g, h \in(2)$;

(ii) $g \in(2)$ and $h \in(3)$; or

(iii) $g, h \in(3)$.

(i) Suppose that

$$
g=x^{\beta_{1}} y^{\varepsilon_{1}} x^{\mu_{1}} \ldots y^{\varepsilon_{n}} x^{\mu_{n}}=x^{\beta_{2}} y^{\delta_{1}} x^{\nu_{1}} \ldots y^{\delta_{m}} x^{\nu_{m}}=h,
$$

where $n, m \geq 1, \beta_{1}, \beta_{2} \geq 0, \mu_{i}$ and $\nu_{i}=0$ or $1, \varepsilon_{i}$ and $\delta_{j}= \pm 1$ $(1 \leq i \leq n, 1 \leq j \leq m)$. then $g h^{-1}$ must HNN-reduce to the trivial word. This forces

$$
\mu_{n}-\nu_{m} \equiv 0 \quad(\bmod 2) \quad \text { which implies that } \mu_{n}=\nu_{m}=0 \text { or } 1,
$$

and $\varepsilon_{n}-\delta_{m}=0$. Since continued reduction must occur we in fact get $m=n$, $\mu_{i}=\nu_{i}$ and $\varepsilon_{i}=\delta_{i}$ for each $i \quad(1 \leq i \leq n)$. Thus $g h^{-1}$ reduces to $x^{\beta_{1}-\beta_{2}}$. Since $G$ is torsion-free, $\beta_{1}=\beta_{2}$.

(ii) and (iii) are similar to (i).

If we count the number of words of length $n$ of each type then, using 
Lemma 1, we find that the growth series are as follow:

$$
\begin{aligned}
& \gamma_{1}=1+\frac{2 t}{1-t}, \\
& \gamma_{2}=\frac{1}{1-t}\left(\frac{1+t}{1-t-2 t^{2}}-1\right), \\
& \gamma_{3}=\frac{2 t^{3}}{1-t}\left(\frac{1+t}{1-t-2 t^{2}}\right)^{2} .
\end{aligned}
$$

Summing $\gamma_{1}+2 \gamma_{2}+\gamma_{3}$ and subtracting $2 t /(1-t)$ which accounts for the subset $I$ gives

$$
\gamma(t)=\frac{1-t-2 t^{3}}{(1-t)(1-2 t)^{2}} .
$$

\section{Further HNN examples}

We now take the presentation

$$
G=\left\langle x, y \mid y^{-1} x^{p} y=x^{p}\right\rangle \quad(p \geq 3)
$$

and calculate the growth series $\gamma(t)$ of $G$ with respect to $X=\{x, y\}$.

For this case the rewrite rules are free reduction together with the following.

RR1 Collect any power of the central element $x^{p}$ to the left.

RR2 If $m>0>n$ and $m+|n|>p$ then replace a word $u x^{n} v x^{m} w$, $u x^{m} v x^{n} w$ (respectively) by $u x^{n+p} v x^{m-p} w, u x^{m-p} v x^{n+p} w$ (respectively).

RR3 If $m>0>n$ and $m+|n|=p$ then replace a word $u x^{n} v x^{m} w$ by $u x^{m} v x^{n} w$.

Certainly if $w \in G$ and $w^{\prime}$ is a word obtained from $w$ after applying any combination of the above rewrite rules then $w=w^{\prime}$ in $G$ and $l\left(w^{\prime}\right) \leq l(w)$. Thus we obtain our proposed collection of normal forms, which consist of the (freely reduced) words in the following lists. We take $p$ even and odd in turn.

$$
p=2 k(k \geq 2)
$$

(1) $x^{\alpha}$ where $\alpha \in \mathbb{Z}$.

$(2)^{ \pm 1} \quad\left(x^{\beta} y^{\varepsilon_{1}} x^{\mu_{1}} \ldots y^{\varepsilon_{n}} x^{\mu_{n}}\right)^{ \pm 1}$ where $n \geq 1, \beta \geq p, \varepsilon_{i}= \pm 1 \quad(1 \leq i \leq n)$ and $\mu_{i} \in\{0,1, \ldots, p-1\} \quad(1 \leq i \leq n)$.

$(3)_{j}^{ \pm 1} \quad(k \leq j \leq p-1)$

$$
\left(x^{\mu_{1 j}} y^{\varepsilon_{1 j}} \ldots x^{\mu_{n_{j} j}} y^{\varepsilon_{n_{j} j}} x^{j} y^{\delta_{1 j}} x^{\gamma_{1 j}} \ldots y^{\delta_{m_{j} j}} x^{\gamma_{m_{j} j}}\right)^{ \pm 1}-\left(x^{j}\right)^{ \pm 1}
$$


where $n_{j}, m_{j} \geq 0, \varepsilon_{i j}= \pm 1 \quad\left(1 \leq i \leq n_{j}\right), \delta_{i j}= \pm 1 \quad\left(1 \leq i \leq m_{j}\right)$,

$$
\begin{aligned}
\mu_{i(p-1)} \in\{0,1, \ldots, p-1\} \quad\left(1 \leq i \leq n_{p-1}\right), & \\
\mu_{i j} \in\{0, \pm 1, \ldots, \pm(p-j-1), p-j, \ldots, j\} & \quad\left(1 \leq i \leq n_{j}, k \leq j<p-1\right), \\
\nu_{i j} \in\{0, \pm 1, \ldots, \pm(p-j), p-j+1, \ldots, j-1\} & \quad\left(1 \leq i \leq m_{j}, k<j \leq p-1\right), \\
\nu_{i k} \in\{0, \pm 1, \ldots, \pm k\} \quad\left(1 \leq i \leq m_{k}\right) . &
\end{aligned}
$$

$$
x^{\mu_{1 k}} y^{\varepsilon_{1}} \ldots x^{\mu_{n k}} y^{\varepsilon_{n}} x^{k} y^{\varepsilon} x^{\nu_{1}} y^{\delta_{1}} \ldots x^{\nu_{m}} y^{\delta_{m}} x^{-k} y^{\gamma_{1}} x^{\lambda_{1}} \ldots y^{\gamma_{l}} x^{\lambda_{l}}
$$

where $n, m, l \geq 0, \varepsilon, \varepsilon_{i}= \pm 1 \quad(1 \leq i \leq n), \delta_{i}= \pm 1 \quad(1 \leq i \leq m), \gamma_{i}= \pm 1$ $(1 \leq i \leq l)$,

$$
\begin{array}{rlrl}
\mu_{i k} & \in\{0, \pm 1, \ldots, \pm(k-1), \pm k\} & (1 \leq i \leq n), \\
\nu_{i} & \in\{0, \pm 1, \ldots, \pm(k-1)\} \quad(1 \leq i \leq m), \\
\lambda_{i} & \in\{0, \pm 1, \ldots, \pm(k-1),-k\} & (1 \leq i \leq l) .
\end{array}
$$

$$
\begin{aligned}
& x^{\nu_{1}} y^{\varepsilon_{1}} \ldots x^{\nu_{n}} y^{\varepsilon_{n}} x^{\nu_{n+1}} \text { where } n \geq 1, \varepsilon_{i}= \pm 1(1 \leq i \leq n), \nu_{i} \in \\
& \{0, \pm 1, \ldots, \pm(k-1)\}(1 \leq i \leq n+1) .
\end{aligned}
$$

The proof of uniqueness in the above list involves straightforward but rather long HNN arguments as in the previous example, so we shall omit it. Given this, it remains to apply Lemma 1 and obtain the growth series,

$$
\begin{aligned}
\gamma_{1}(t) & =\frac{1+t}{1-t}, \\
\gamma_{2}(t) & =\frac{t^{p}}{1-t}\left(\frac{1+t}{1-t-P_{(p-1)}(t)}-1\right), \\
\gamma_{3_{j}}(t) & =\frac{t^{j}(1+t)^{2}}{\left.1-t-P_{(j-1)}(t)\right)\left(1-t-P_{j}(t)\right)}-t^{j} \quad(k<j \leq p-1), \\
\gamma_{3_{k}}(t) & =\frac{t^{k}(1+t)^{2}}{\left(1-t-P_{k}(t)\right)(1-t-Q(t))}-t^{k}, \\
\gamma_{4}(t) & =\frac{2(1+t)^{3} t^{p+1}}{\left(1-t-P_{k}(t)\right)^{2}(1-t-Q(t))}, \\
\gamma_{5}(t) & =\left(1+\sum_{i=1}^{k-1} 2 t^{i}\right)\left(\frac{1+t}{1-t-Q(t)}-1\right),
\end{aligned}
$$

where

$$
P_{j}(t)=\frac{4 t^{2}\left(1-t^{p-j-1}\right)+2 t^{p-j+1}\left(1-t^{2 j-p+1}\right)}{1-t} \quad(k \leq j \leq p-1),
$$


and

$$
Q(t)=\frac{4 t^{2}\left(1-t^{k-1}\right)}{1-t}
$$

Summing

$$
\gamma_{1}(t)+2 \gamma_{2}(t)+2 \sum_{i=k}^{p-1} \gamma_{3 i}(t)+\gamma_{4}(t)+\gamma_{5}(t)
$$

we obtain the following result.

Proposition 1. If $G=\left\langle x, y \mid y^{-1} x^{2 k} y=x^{2 k}\right\rangle(k \geq 2)$ then

$$
\begin{aligned}
\gamma(t)= & \frac{2 t^{2 k}(t+1)}{2 t^{2 k+1}-t^{2}-2 t+1} \\
& +\sum_{i=1}^{k-1} \frac{2 t^{k+3 i}\left(1-t^{2}\right)^{2}}{\left(2 t^{k+2 i+1}+2 t^{k+2}-3 t^{i+2}-2 t^{i+1}+t^{i}\right)\left(2 t^{k+2 i+2}+2 t^{k+1}-3 t^{i+2}-2 t^{i+1}+t^{i}\right)} \\
& +\frac{(1+t)\left[\left(1+t-2 t^{k}\right)\left(2 t^{k+1}-3 t+1\right)^{2}+2 t^{2 k+1}(1-t)^{3}+2 t^{k}(1-t)^{2}\left(2 t^{k+1}-3 t+1\right)\right.}{\left(2 t^{k+1}-3 t+1\right)^{2}\left(4 t^{k+1}-3 t^{2}-2 t+1\right)}
\end{aligned}
$$

We turn now to the list of minimal forms for $p$ odd.

$$
p=2 k+1 \quad(k \geq 1)
$$

(1) $x^{\alpha}$ where $\alpha \in \mathbb{Z}$.

$(2)^{ \pm 1} \quad\left(x^{\beta} y^{\varepsilon_{1}} x^{\mu_{1}} \ldots y^{\varepsilon_{n}} x^{\mu_{n}}\right)^{ \pm 1}$ where $n \geq 1, \beta \geq p, \varepsilon_{i}= \pm 1 \quad(1 \leq i \leq n)$ and $\mu_{i} \in\{0,1, \ldots, 2 k\} \quad(1 \leq i \leq n)$.

$(3)_{j}^{ \pm 1}$

$(k+1 \leq j \leq p-1) \quad\left(x^{\mu_{1 j}} y^{\varepsilon_{1 j}} \ldots x^{\mu_{n_{j} j}} y^{\varepsilon_{n_{j} j}} x^{j} y^{\delta_{1 j}} x^{\nu_{1 j}} \ldots y^{\delta_{m_{j} j}} x^{\nu_{m_{j} j}}\right)^{ \pm 1}-\left(x^{j}\right)^{ \pm 1}$,

where

$$
\begin{gathered}
n_{j}, m_{j} \geq 0, \quad \varepsilon_{i j}= \pm 1 \quad\left(1 \leq i \leq n_{j}\right), \quad \delta_{i j}= \pm 1\left(1 \leq i \leq m_{j}\right), \\
\mu_{i(p-1)} \in\{0,1, \ldots, p-1\} \quad\left(1 \leq i \leq n_{p-1}\right), \\
\mu_{i j} \in\{0, \pm 1, \ldots, \pm(p-j-1), p-j, \ldots, j\} \\
\nu_{i j} \in\{0, \pm 1, \ldots, \pm(p-j), p-j+1, \ldots, j-1\} \\
\gamma_{i(k+1)} \in\{0, \pm 1, \ldots, \pm k\} \quad\left(1 \leq i \leq n_{j}\right) \quad(k \leq j<p-1), \\
\begin{array}{c}
(4) \quad x^{\nu_{1}} y^{\varepsilon_{1}} \ldots x^{\nu_{n}} y^{\varepsilon_{n}} x^{\nu_{n+1}} \quad \text { where } n \geq 1, \varepsilon_{i}= \pm 1 \quad\left(1 \leq i \leq m_{j}\right) \quad(k+1<j \leq p-1), \\
\{0, \pm 1, \ldots, \pm k\} \quad(1 \leq i \leq n) .
\end{array}
\end{gathered}
$$


Again the uniqueness arguments involve standard HNN theory. The corresponding growth series are

$$
\begin{aligned}
& \gamma_{1}(t)=\frac{1+t}{1-t} \\
& \gamma_{2}(t)=\frac{t^{p}}{1-t}\left(\frac{1+t}{1-t-P_{(p-1)}(t)}-1\right), \\
& \gamma_{3_{j}}(t)=\frac{t^{j}(1+t)^{2}}{\left(1-t-P_{(j-1)}(t)\right)\left(1-t-P_{j}(t)\right)}-t^{j} \quad(k+1 \leq j \leq p-1),
\end{aligned}
$$

and

$$
\gamma_{4}(t)=\left(1+\sum_{i=1}^{k} 2 t^{k}\right)\left(\frac{1+t}{1-t-P_{k}(t)}-1\right),
$$

where

$$
P_{i}(t)=\frac{4 t^{2}\left(1-t^{2 k-i}\right)+2 t^{2 k-i+2}\left(1-t^{2 i-2 k}\right)}{1-t} \quad(k \leq i \leq p-1) .
$$

Summing

$$
\gamma_{1}(t)+2 \gamma_{2}(t)+2 \sum_{j=k+1}^{p-1} \gamma_{3_{j}}(t)+\gamma_{4}(t)
$$

gives the next result.

Proposition 2. If $G=\left\langle x, y \mid y^{-1} x^{2 k+1} y=x^{2 k+1}\right\rangle(k \geq 1)$ then

$$
\begin{aligned}
\gamma(t) & =\frac{2 t^{2 k+1}(1+t)}{2 t^{2 k+2}-t^{2}-2 t+1} \\
+ & \sum_{i=1}^{k} \frac{2 t^{k+i}(1+t)^{2}(1-t)^{2}}{\left(2 t^{k+i-1}+2 t^{k-i+3}-3 t^{2}-2 t+1\right)\left(2 t^{k+i+2}+2 t^{k-i+2}-3 t^{2}-2 t+1\right)} \\
& +\frac{\left(1+t-2 t^{k+1}\right)(1+t)}{4 t^{k+2}-3 t^{2}-2 t+1} .
\end{aligned}
$$

Notation. We write $\left(a_{0}, a_{1}, \ldots, a_{m}\right)$ to denote $\sum_{i=0}^{m} a_{i} t^{i}$.

EXAMPLES. (i) $G=\left\langle x, y \mid y^{-1} x^{4} y=x^{4}\right\rangle$ has growth series

$$
\gamma(t)=\frac{(1,-4,-3,20,8,-36,-12,22,-16,-40,-12)}{(1-t)\left(1-t-4 t^{2}\right)\left(1-2 t-2 t^{3}\right)\left(1-2 t-2 t^{2}\right)^{2}} .
$$


(ii) $G=\left\langle x, y \mid y^{-1} x^{6} y=x^{6}\right\rangle$ has growth series $\gamma(t)=$ $\frac{(1,-6,3,26,8,-72,-102,-16,192,294,236,28,32,148,292,328,280,208,88,32)}{(1-t)\left(1-t-4 t^{2}-4 t^{3}\right)\left(1-2 t-2 t^{2}-2 t^{4}\right)\left(1-2 t-2 t^{3}-2 t^{5}\right)\left(1-2 t-2 t^{2}-2 t^{3}\right)^{2}}$.

(iii) $G=\left\langle x, y \mid y^{-1} x^{3} y=x^{3}\right\rangle$ has growth series

$$
\gamma(t)=\frac{(1+t)^{2}(1-2 t)\left(1+t+2 t^{3}\right)}{(1-t)\left(1-t-4 t^{2}\right)\left(1-t-2 t^{2}-2 t^{3}\right)} .
$$

(iv) $G=\left\langle x, y \mid y^{-1} x^{5} y=x^{5}\right\rangle$ has growth series

$$
\gamma(t)=\frac{(1+t)^{2}(1,-2,-5,2,12,14,-2,0,8,20,8,8)}{(1-t)\left(1-t-4 t^{2}-4 t^{3}\right)\left(1-t-4 t^{2}-2 t^{3}-2 t^{4}\right)\left(1-t-2 t^{2}-2 t^{3}-2 t^{4}-2 t^{5}\right)} .
$$

REMARK. Further investigation strongly suggests that the degrees of the numerator and denominator coincide and what the general form of the denominator will be. If $d$ denotes the degree of the denominator then

$$
d=1+\sum_{i=k+1}^{p} i=\frac{1}{8}\left(3 p^{2}+4 p+9\right) \quad(p=2 k+1, k \geq 1)
$$

and

$$
d=1+p+\sum_{i=l}^{p-1} i=\frac{1}{8}\left(3 p^{2}+6 p+8\right) \quad(p=2 l, l \geq 2)
$$

To complete the picture, if $p=0$ then $\gamma(t)=(1+t) /(1-t)$, and if $p=1$ then $\gamma(t)=((1+t) /(1-t))^{2}$. So the formulae above apply except when $p=2$.

\section{Some amalgamated free products}

Finally we compute the growth series $\gamma(t)$ with respect to $X=\{x, y\}$ for the group presentation

$$
G=\left\langle x, y \mid x^{p}=y^{p}\right\rangle \quad(p \geq 1) .
$$

The rewrite rules for this case are the following.

RR1 Collect any powers of the central elements $x^{p}, y^{p}$ to the left and write as a power of $x^{p}$.

RR2 Replace a word $u x^{p} v z^{-l} w, u x^{-p} v z^{l} w$ (respectively) ( $z=x$ or $y$, $l \in \mathbb{N})$ by $u v z^{p-l} w, u v z^{l-p} w$ (respectively).

RR3 If $m+n>p$ replace a word $u z_{1}^{-m} v z_{2}^{n} w, u z_{1}^{n} v z_{2}^{-m} w$ (respectively), $\left(z_{1}\right.$ and $z_{2}=x$ or $y$ ) by $u z_{1}^{p-m} v z_{2}^{n-p} w, u z_{1}^{n-p} v z_{2}^{p-m} w$ (respectively).

RR4 Replace a word $u z_{1}^{l} v z_{2}^{l-p} w$ by $u z_{1}^{l-p} v z_{2}^{l} w \quad\left(z_{1}\right.$ and $z_{2}=x$ or $\left.y\right)$. 
In what follows, $A=\langle x\rangle \backslash\{e\}, B=\langle y\rangle \backslash\{e\}$ and

$$
{ }_{-a} A \pi B_{b} \quad(a, b \in \mathbb{N})
$$

denotes the subset of $A \pi B$ of words that involve no power of $x$ or $y$ lower than $-a$ or higher than $b$. In this case we get

$$
\begin{gathered}
\gamma_{A}=\gamma_{B}=\frac{t-t^{a+1}}{1-t}+\frac{t-t^{b+1}}{1-t}=\frac{t\left(2-t^{a}-t^{b}\right)}{1-t}, \\
\frac{\left(1+\gamma_{A}\right)\left(1+\gamma_{B}\right)}{1-\gamma_{A} \gamma_{B}}=\frac{1+t-t^{a+1}-t^{b+1}}{1-3 t+t^{a+1}+t^{b+1}},
\end{gathered}
$$

and

$$
\frac{1+\gamma_{B}}{1-\gamma_{A} \gamma_{B}}=\frac{1-t}{t^{a+1}+t^{b+1}+1-3 t}
$$

Given a freely reduced word in $F(X)$, once we have applied the rewrite rules RR1 and RR2 either this gives the normal form

$(1)^{ \pm 1}\left(x^{p}\right)_{1}^{k} A \pi B_{p-1}(k \in \mathbb{N})$ together with their inverses $(k<0)$, or an alternating word $\left({ }_{-[p / 2]} A \pi B_{p-1}\right)^{ \pm 1}$. Applying RR3 and RR4 to this word gives one of the following normal forms.

$(2)_{j}^{ \pm 1} \quad([p / 2]<j \leq p-1)_{j-p} A \pi_{r} B_{j-1}\left(x^{j}\right)_{j-p+1} A_{r} \pi B_{j}$ and similarly with $y^{j}$, and inverses of both.

(3) $-[p-1 / 2] A \pi B_{[p-1 / 2]}$.

When $p=2 k$ is even we have to take into account the cases when $k$ is the highest power to occur and $-k$ does or does not occur. The normal forms are

$(4)^{ \pm 1}-k+1=\pi_{r} B_{k-1}\left(x^{k}\right)_{-k+1} A_{r} \pi B_{k}$ and similarly with $y^{k}$, and inverses of both.

(5)

$$
{ }_{-k} A \pi_{r} B_{k-1}\left(x^{-k}\right)_{-k+1} A_{r} \pi_{r} B_{k-1}\left(x^{k}\right)_{-k+1} A_{r} \pi Y_{k}
$$

and

$$
{ }_{-k} A \pi_{l} B_{k-1}\left(y^{-k}\right)_{-k+1} A_{l} \pi_{r} B_{k-1}\left(x^{k}\right)_{-k+1} A_{r} \pi B_{k}
$$

together with the forms obtained on interchanging $x$ and $y$.

To prove uniqueness a simple case-by-case analysis shows that two distinct normal forms cannot have the same image under both the natural maps

$$
\begin{aligned}
& \phi_{1}: G \rightarrow D=\left\langle x, y \mid x^{p}=y^{p}=1\right\rangle \\
& \phi_{2}: G \rightarrow Z=\langle x, y \mid x=y\rangle
\end{aligned}
$$

and so they represent different elements of $G$, as $\operatorname{Ker} \phi_{1} \cap \operatorname{Ker} \phi_{2}=\{e\}$. 
We have, using Lemma 2 and the remarks made earlier in this section, that the corresponding growth series are as follows.

$$
\begin{aligned}
\gamma_{1}(t)= & \frac{t^{p}\left(1-t^{p}\right)}{1-2 t+2 t^{p+1}-t^{2 p}}, \\
\gamma_{2_{j}}(t)= & \frac{t^{j}(1-t)^{2}}{\left(1-3 t+t^{j}+t^{p-j+1}\right)\left(1-3 t+t^{j+1}+t^{p-j}\right)}, \\
\gamma_{3}(t)= & \frac{1+t-2 t^{k+1}}{1-3 t+2 t^{k+1}} \quad(p=2 k+1), \\
& \frac{1+t-2 t^{k}}{1-3 t+2 t^{k}} \quad(p=2 k), \\
\gamma_{4}(t)= & \frac{t^{k}(1-t)^{2}}{\left(1-3 t+2 t^{k}\right)\left(1-3 t+t^{k}+t^{k+1}\right)}, \\
\gamma_{5}(t)= & \frac{t^{p}(1-t)^{3}}{\left(1-3 t+t^{k}+t^{k+1}\right)^{2}\left(1-3 t+2 t^{k}\right)} .
\end{aligned}
$$

For $p$ odd we must form the sum $2 \gamma_{1}(t)+4 \sum_{j=k+1}^{2 k} \gamma_{2_{j}}(t)+\gamma_{3}(t)$, and for $p$ even the sum is $2 \gamma_{1}(t)+4 \sum_{j=k+1}^{p-1} \gamma_{2 j}(t)+\gamma_{3}(t)+4 \gamma_{4}(t)+2 \gamma_{5}$.

Proposition 3. Let $G=\left\langle x, y \mid x^{p}=y^{p}\right\rangle$ where $p \geq 1$.

(i) If $p=2 k+1 \quad(k \geq 0)$ then the growth series of $G$ is

$$
\begin{aligned}
\gamma(t)= & \frac{2 t^{p}}{t^{p}-2 t+1}+\frac{1+t-2 t^{k+1}}{1-3 t+2 t^{k+1}} \\
& +\sum_{j=k+1}^{2 k} \frac{4 t^{3 j}(1-t)^{2}}{\left(t^{2 j}-3 t^{j+1}+t^{j}+t^{p+1}\right)\left(t^{2 j+1}-3 t^{j+1}+t^{j}+t^{p}\right)} .
\end{aligned}
$$

(ii) If $p=2 l \quad(l \geq 1)$ then the growth series of $G$ is

$$
\begin{aligned}
\gamma(t) & =\frac{2 t^{p}}{t^{p}-2 t+1}+\frac{1+t-2 t^{l}}{1-3 t+2 t^{l}} \\
& +\sum_{j=l}^{p-1} \frac{4 t^{3 j}(1-t)^{2}}{\left(t^{2 j}-3 t^{j+1}+t^{j}+t^{p+1}\right)\left(t^{2 j+1}-3 t^{j+1}+t^{j}+t^{p}\right)} \\
& +\frac{4 t^{l}(1-t)^{2}}{\left(1-3 t+2 t^{l}\right)\left(1-3 t+t^{l}+t^{l+1}\right)}+\frac{2 t^{p}(1-t)^{3}}{\left(1-3 t+t^{l}+t^{l+1}\right)^{2}\left(1-3 t+2 t^{l}\right)} .
\end{aligned}
$$

\section{EXAMPLES.}

$$
G=\left\langle x, y \mid x^{2 k}=y^{2 k}\right\rangle(k \geq 1) .
$$


(i) $p=2 k=2: \quad \gamma(t)=\frac{(1+t)^{2}}{(1-t)^{2}}$.

(ii) $p=4: \gamma(t)=\frac{(1+t)\left(1-3 t+t^{2}+3 t^{3}-5 t^{4}+3 t^{5}+3 t^{6}+t^{7}\right)}{(1-t)\left(1-2 t-t^{2}\right)^{2}\left(1-t-t^{2}-t^{3}\right)}$.

(iii) $p=6: \gamma(t)=\frac{(1+t)(1,-5,4,7,2,-6,-22,6,8,-2,-14,-14,-15,-9,-4,1)}{(1-t)\left(1-2 t-2 t^{2}-t^{3}\right)^{2}\left(1-2 t-t^{2}-t^{3}-t^{4}\right)\left(1-t-t^{2}-t^{3}-t^{4}-t^{5}\right)}$.

(iv) $p=8: \gamma(t)=(1+t) V(t)\left[(1-t)\left(1-2 t-2 t^{2}-2 t^{3}-t^{4}\right)^{2}\left(1-2 t-2 t^{2}-t^{3}-t^{4}-t^{5}\right)\right]^{-1}$

$$
\times\left[\left(1-2 t-t^{2}-t^{3}-t^{4}-t^{5}-t^{6}\right)\left(1-t-t^{2}-t^{3}-t^{4}-t^{5}-t^{6}-t^{7}\right)\right]^{-1},
$$

where $V(t)$ is a polynomial with integer coefficients and of degree 26 .

EXAMPLES.

$$
G=\left\langle x, y \mid x^{2 k+1}=y^{2 k+1}\right\rangle(k \geq 0) .
$$

(i) $p=2 k+1=1: \quad \gamma(t)=\frac{1+t}{1-t}$.

(ii) $p=3: \quad \gamma(t)=\frac{(1+t)\left(1-t+t^{2}-2 t^{3}\right)}{(1-t)(1-2 t)\left(1-t-t^{2}\right)}$.

(iii) $p=5$ :

$$
\gamma(t)=\frac{(1+t)(1,-3,3,3,-6,2,5,2,2)}{(1-t)\left(1-2 t-2 t^{2}\right)\left(1-2 t-t^{2}-t^{3}\right)\left(1-t-t^{2}-t^{3}-t^{4}\right)} .
$$

(iv) $p=7$ :

$$
\begin{aligned}
\gamma(t)= & (1+t) W(t)\left[(1-t)\left(1-2 t-2 t^{2}-2 t^{3}\right)\left(1-2 t-2 t^{2}-t^{3}-t^{4}\right)\right]^{-1} \\
& \times\left[\left(1-2 t-t^{2}-t^{3}-t^{4}-t^{5}\right)\left(1-t-t^{2}-t^{3}-t^{4}-t^{5}-t^{6}\right)\right]^{-1},
\end{aligned}
$$

where $W(t)$ has integer coefficients and has degree 19 .

REMARK. As in the previous section, a pattern is emerging. If $d$ denotes the degree of the numerator (= degree of denominator) of $\gamma(t)$ then $d=$ $(1 / 8)\left(3 p^{2}+5\right)$ for $p$ odd, and $d=(1 / 8)\left(3 p^{2}+2 p+8\right)$ for $p$ even and at least 4.

ADDED IN PROOF. The first named author and D. J. Collins have now calculated the growth series of the group $\left\langle x, y \mid y^{-1} x y=x^{2}\right\rangle$ with respect to $\{x, y\}$, and $\mathbf{M}$. Brazil has independently shown this group to have national growth series.

ACKNOWLeDgements. We thank Douglas Woodall for his comments in connection with this work and Dave Wood for his help with some of the calculations.

\section{References}

[1] M. Benson, 'On the rational growth of virtually nilpotent groups,' Combinatorial Group Theory and Topology, Princeton Studies no. 111, pp. 185-196 (Princeton University Press, Princeton 1987).

[2] N. Billington, The growth of stratified groups (PhD thesis, La Trobe University, 1986). 
[3] J. W. Cannon, 'The growth of the closed surface groups and compact hyperbolic groups,' preprint.

[4] V. E. Govorov, 'On the dimension of graded algebras,' Mat Zametki 14 (1973), 209-216 (translation: Math. Notes 14 (1973), 678-682).

[5] R. I. Grigorchuk, 'On Milnor's problem of group growth,' Dokl. Akad. Nauk SSSR 271: 1(1983) (translation: Soviet Math. Dokl. 28 (1983), 23-26).

[6] J. R. J. Groves and G. C. Smith, 'Rewriting systems and soluble groups,' Bath Comp Sci Tech Report no. 19 (1989).

[7] F. Grünewald, seminar at Berkeley, January 1989.

[8] N. D. Gupta and S. Sidki, 'On the Burnside problem for periodic groups,' Math. Z. 182 (1983), 385-388.

[9] D. L. Johnson, 'Rational growth of wreath products,' in Groups St. Andrews 1989 (ed. C. M. Campbell and E. F. Robertson), pp. 309-315, Cambridge Univ. Press, 1991.

[10] P. Le Chedanec, Canonical forms in finitely presented algebras (Pitman, London 1986).

[11] M. Lorenz, Gel' fand-Kirillov dimension and Poincaré series (Lecture Notes, De Kalb 1987).

[12] R. C. Lyndon and P. E. Schupp, Combinatorial Group Theory (Springer, Berlin 1977).

[13] W. Magnus, Noneuclidean tessellations and their groups (Academic Press, New YorkLondon, 1974).

[14] J. Milnor, 'A note on curvature and the fundamental group,' J. Differential Geom. 2 (1968), 1-7.

[15] N. J. Smythe, 'Growth functions and Euler series,' Invent. Math. 77 (1984), 517-531.

\section{University of Nottingham}

University Park

Nottingham NG7 2RD

U.K. 